\title{
Management of Dyed Area in Hydrophilic Paint-gun Injury of the Hand
}

\author{
Hyung Mook Kim, Sae Hwi Ki, Sang Hwan Lee \\ Department of Plastic Surgery, Inha University Hospital, Inha University School of Medicine, Incheon, Korea
}

\begin{abstract}
The authors report a case of hydrophilic high-pressure paint-gun injury of the hand which differed from typical highpressure injection injuries. The patient was managed conservatively for four days after trauma because the injected agent was initially mistaken for a totally water-soluble material. As a result, the injected hydrophilic paint spread up to the upper arm. Foreign materials observed in the hand were debrided but pigmented areas in the left forearm and upper arm were retained based on considerations of functional limitation. One year after surgery, the patient had no functional complications though paint was still observed in the forearm.
\end{abstract}

Keywords: Hand, Hand injuries, Injection, Paint

\section{Introduction}

Although the incidence of hand injuries caused by high-pressure injection devices is not high, compartment syndrome and soft tissue necrosis can cause severe post-injury disabilities such as loss of hand function, and amputation rates as high as $48 \%$ have been reported [1]. Treatments for these injuries are early adequate debridement and infection control, and additional secondary reconstruction when needed. It has been reported complete removal of high-pressure sprayed material is of utmost importance to prevent complications $[2,3]$. The removal of injected foreign substances is obviously necessary but guidelines are lacking for the treatment for pigmented sites, and the removal of pigmented parts can lead to severe functional defects. We experienced a case of a high-pressure hydrophilic paint-gun injury. Due to delayed treatment, foreign materials of paint in the left hand were removed by debridement but pigmented regions in the left forearm and upper arm were treated conservatively. A good result was obtained, and thus, we share details of this case.

\section{Case report}

A 57-year-old male visited our emergency department with a high-pressure (pressure $55-85 \mathrm{lb} / \mathrm{in}^{2}$ ) paint-gun injection injury on the dorsum of his left hand. He had no specific medical history. When the patient first visited the emergency department, pain and swelling were not severe and soft tissue was nearly intact (Fig. 1). Because the patient reported that the injected material was water-soluble, massive irrigation was performed in the emergency department and he was then discharged and instructed to visit the outpatient department for follow up. Three days after discharge, the patient visited the outpatient department with complaints of severe swelling, a heating sensation, and pain, and it was observed that the injected paint had spread to

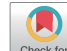

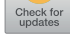 \\ Case Report \\ Received: June 19, 2018 \\ Revised: August 16, 2018 \\ Accepted: August 17, 2018

\section{Corresponding author:} \\ Sang Hwan Lee \\ Department of Plastic Surgery, Inha University \\ Hospital, Inha University School of Medicine, \\ 27 Inhang-ro, Jung-gu, Incheon 22332, Korea \\ Tel: +82-10-3761-7882 \\ Fax +82-32-890-2918 \\ E-mail: sangwind@hanmail.net
}

This is an Open Access article distributed under the terms of the Creative Commons Attribution Non-Commercial License (http://creativecommons.org/licenses/by-nc/4.0/) which permits unrestricted non-commercial use, distribution, and reproduction in any medium, provided the original work is properly cited.

๑) 2018 Korean Wound Management Society 


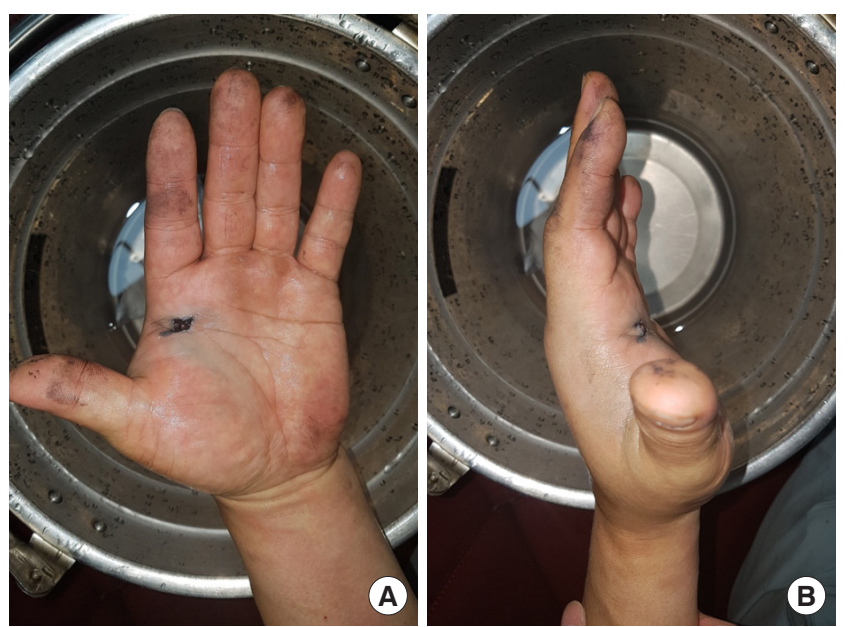

Fig. 1. Clinical photographs at the emergency room. A 57-year-old male visited our emergency department with a high-pressure hydrophilic paint-gun injection injury on the dorsum of his left hand. the upper left arm (Fig. 2). Mild active movement limitation was observed but circulation and sensation were intact. We asked the patient to identify the components of the injected paint, and found that it was not water-soluble but hydrophilic paint (Table 1). Simple radiographs revealed the presence of foreign material in the first web space but not in the upper arm, with no evidence of bone destruction. To evaluate the amount of injected paint and its spread, a computerized tomography scan was requested and it revealed the paint had spread around the thenar muscle layer on volar and dorsum regions of first web space (Fig. 2). A general intoxication test performed at the time showed high erythrocyte sedimentation rate (ESR) and C-reactive protein (CRP) but a normal blood glucose level. Blood culture and wound smear test were negative.
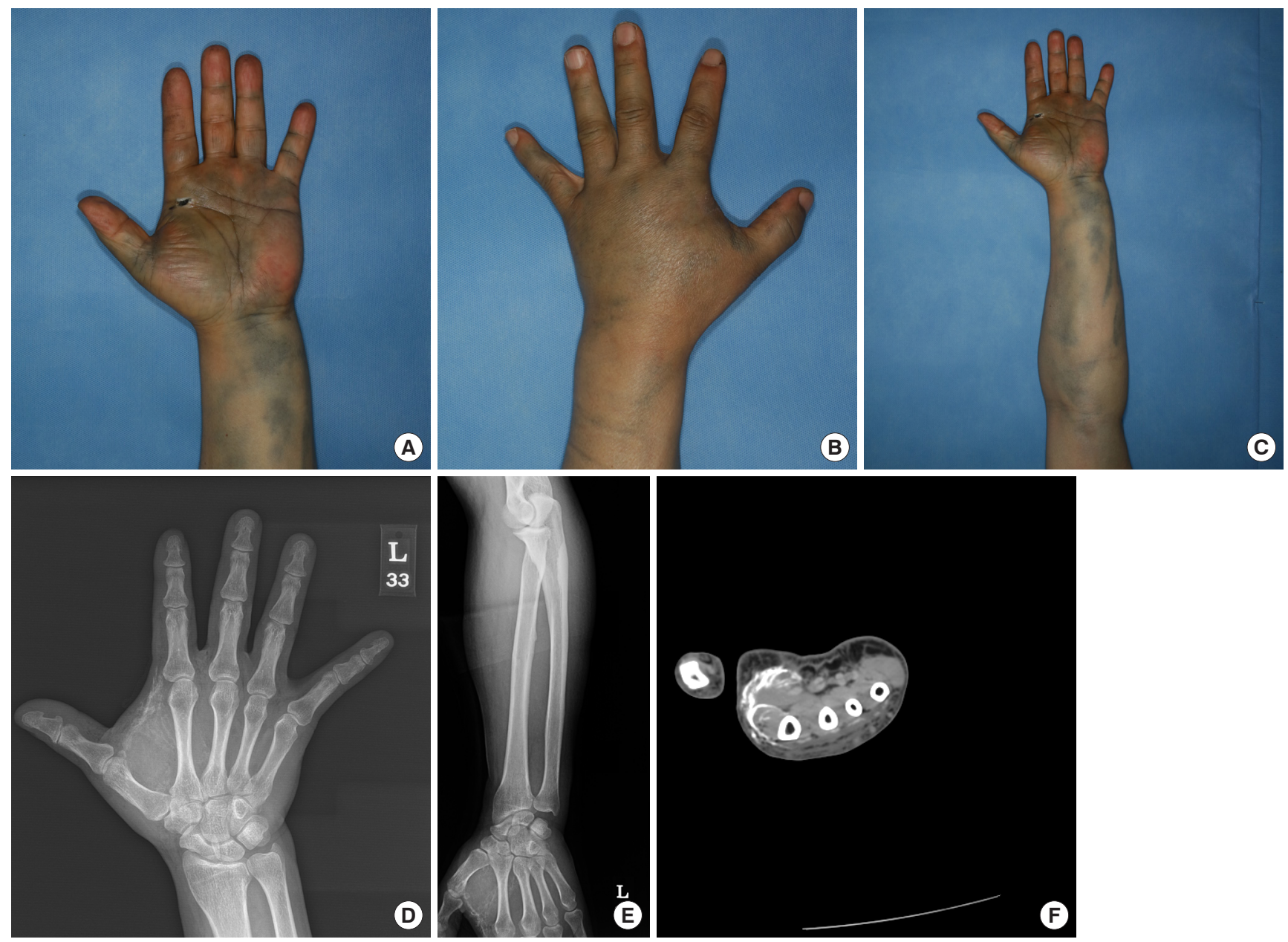

Fig. 2. At 3 days after the injury, clinical photographs, X-ray and computed tomography. It was observed the injected paint had spread to the upper left arm (A-C), X-ray of the left hand revealed the presence of foreign material in the first web space and left foream (D, E), and computed tomography revealed the paint had spread around the thenar muscle layer on volar and dorsum regions of first web space $(F)$. 
Surgery was performed for incision and drainage. Under general anesthesia, a zig-zag shaped skin incision was placed

Table 1. Paint components injected by high pressure gun

\begin{tabular}{lc}
\hline Component & Content (\%) \\
\hline Water & $31-40$ \\
Hydroxylethyl cellulose ether & $0-1$ \\
Propanoio acid & $1-10$ \\
Talc & $21-30$ \\
limestone & $1-10$ \\
\hline
\end{tabular}

on the first web space. Operation findings showed the thenar muscle was pigmented by the paint and abundant calcareous materials (Fig. 3). Foreign materials were removed and massive irrigation was performed. Pigment removal proved difficult and massive irrigation achieved little discernable improvement. Because the excision of pigmented tissue could have resulted in many functional and cosmetic problems, pigmented parts were peeled off as much as possible and tissue excision was minimized. After surgery, treatment consisted of intravenous antibiotics administration and simple wound dressing.
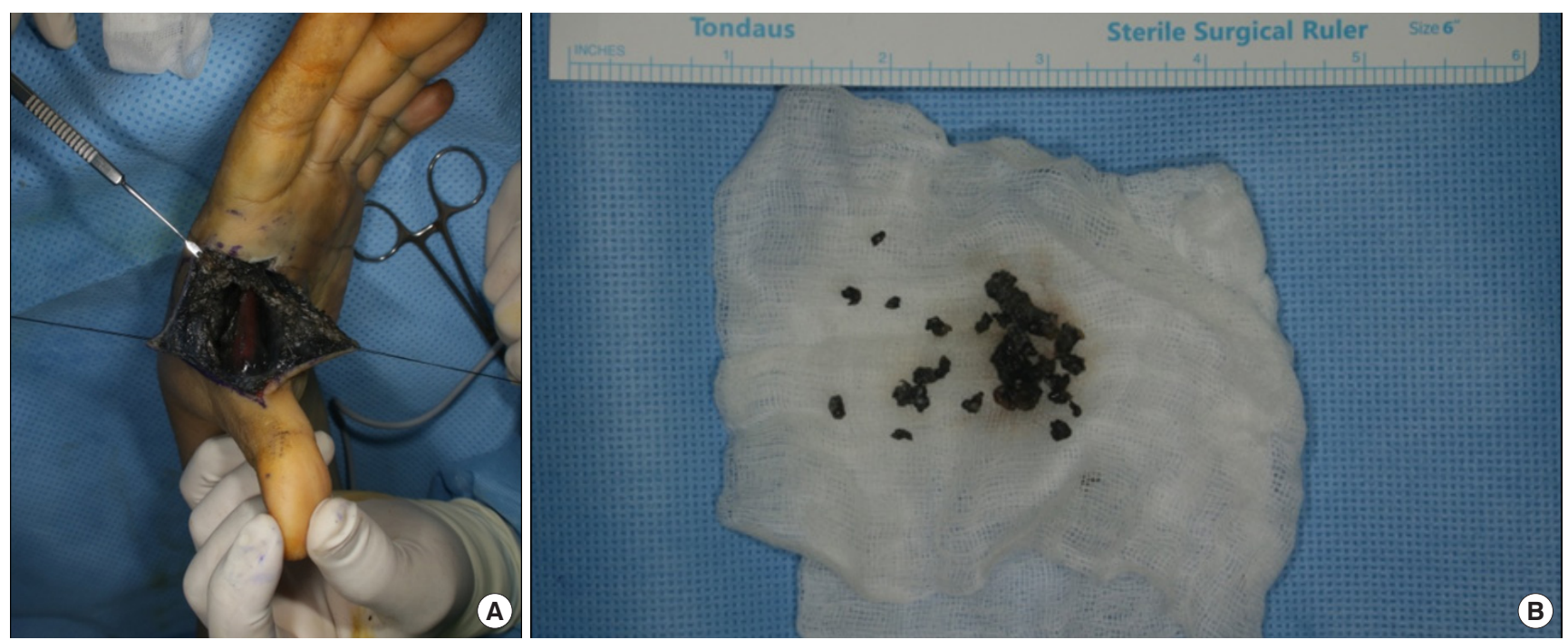

Fig. 3. Intraoperative clinical photographs. Thenar muscle was pigmented by the paint (A) and calcareous materials of first web space were removed (B).
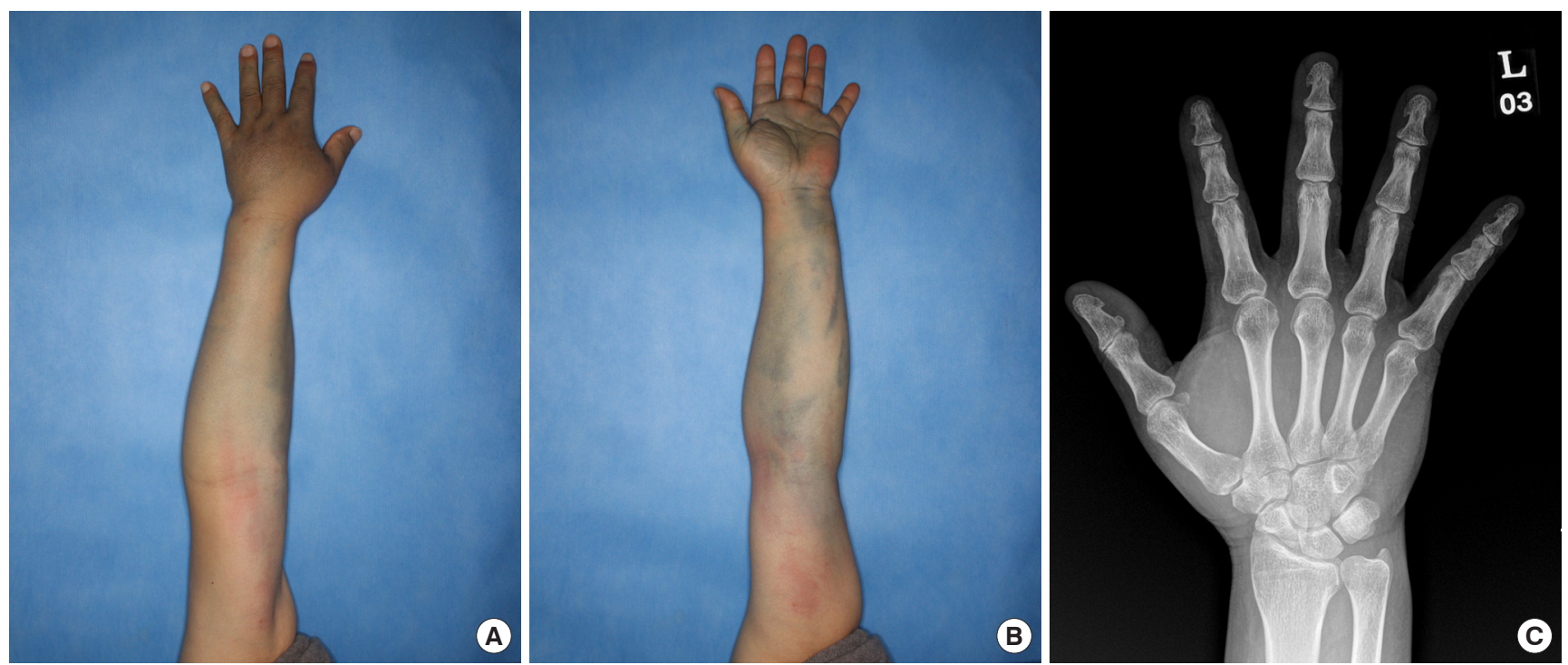

Fig. 4. Clinical photographs and X-ray, postoperative 1 year. Dyed area of injected paint had faded but remained obvious (A, B) and no foreign bodies were observed on $\mathrm{x}$-ray $(\mathrm{C})$. 
At one week after surgery, loss of pain and full range of active movement of the hand were observed. Intravenous antibiotics were then switched to oral antibiotics, and subsequently, symptom improvement was maintained and CRP normalized. The patient was discharged at 10 days after surgery. At one year after the operation, pigmented areas had faded but remained obvious, joint motion was normal, and hand function had totally recovered without any notable complications (Fig. 4).

\section{Discussion}

A substance injected into the hand by high-pressure can spread to other parts of the affected limb, for example, in injection injuries to the thumb or little finger, the problematic substance can spread to the forearm through radial or ulnar bursae [4]. It has also been known that high-pressure injuries to fingers are likely to have poorer prognoses than palm injuries, because fingers have less free space [5]. In our case, injection injury was to the left palm and subjective symptoms were not severe. The paint was injected into the space between the subcutaneous layer and thenar muscle and this resulted in less initial foreign body sensation and soft tissue complications than would be expected for a finger injury. In addition, emergency room medical staff considered the injected paint as something similar to water because the patient said it was a water-soluble paint, and in general, injuries by such water-soluble materials are treated conservatively [6]. However, the injected paint in our case was a hydrophilic paint that could not be completely dissolved in water, containing various substances including calcareous materials (Table 1). These were detected as foreign materials by radiologic examination. As a result of the treatment delay, the pigment had spread to the upper arm and had pigmented so much tissue that its complete removal was not possible.

The prognostic factors of hand injuries caused by high pressure injection are injection pressure and the amount and nature of the material injected. It has been reported when the injection pressure exceeds $7,000 \mathrm{lb} / \mathrm{in}^{2}$, the amputation rate is around $100 \%[4,6]$, and obviously, the greater the amount injected, the poorer the prognosis [6,7]. The effects of paint components on prognoses have not been well-established because of the low incidence of paint-gun injuries. Nevertheless, oil-based paint injection injuries are considered to have poorer prognoses than water-based paint injuries. Furthermore, oilbased paint injection can induce severe inflammatory reactions $[4,6,8-10]$.
In our case, the operating pressure of the paint gun, which was being used to seal cracks during a waterproofing operation, was only $55-85 \mathrm{lb} / \mathrm{in}^{2}$. Furthermore, the paint was injected into the palmar area of the left hand. This meant it could spread into a relatively large space, which prevented excessive mechanical stimulation of surrounding tissues. In addition, foreign body reactions in pigmented areas were low in our patient. Because the amputation rate of high pressure injection injury to the hand is high, such injuries are treated as surgical emergencies requiring immediate decompression by through removal of offending material from tissues to preserve optimal function [3]. However, the removal of all pigmented areas by a hydrophilic paint may lead to unnecessary cosmetic and functional problems. We report a case of high pressure injection of a hydrophilic paint into the palmar area of the left hand. The patient was not treated as a surgical emergency because he said it was a water-soluble and the paint spread to the upper arm. Injected material was removed by debridement and cleansing as much as possible in the hand but pigmented area to the upper arm was treated conservatively to minimize functional and cosmetic defects. The patient has been successfully treated without functional complications.

\section{Conflict of interest}

No potential conflict of interest relevant to this article.

\section{References}

1. Schoo MJ, Scott FA, Boswick JA Jr. High-pressure injection injuries of the hand. J Trauma 1998;20:229-38.

2. Failla JM, Linden MD. The acute pathologic changes of paint injection injury and correlation to surgical treatment: a report of two cases. J Hand Surg Am 1997;221:156-9.

3. Valentino M, Rapisarda V, Fenga C. Hand injuries due to high pressure injection devices for painting in shipyards: circumstances, management, and outcome in twelve patients. Am J Ind Med 2003;43:539-42.

4. Schnall SB, Mirzayan R. High-pressure injection injuries to the hand. Hand Clin 1999;15:245-8.

5. Neal NC, Burke FD. High-pressure injection injuries. Injury 1991;122:467-70.

6. Amsdell SL, Hammert WC. High-pressure injection injuries in the hand: current treatment concepts. Plast Reconstr Surg 2013;132:586-91.

7. Couzens G, Burke FD. Veterinary high pressure injection in- 
Kim HM et al.

Hydrophilic paint-gun injury

juries with inoculations for larger animals. J Hand Surg Br 1995;20:497-9.

8. Lewis HG, Clarke P, Kneafsey B, et al. A 10-year review of high-pressure injection injuries to the hand. J Hand Surg $\mathrm{Br}$ 1998;23:479-81.

9. Mirzayan R, Schnall SB, Chon JH, et al. Culture results and amputation rates in high pressure paint gun injuries of the hand. Orthopedics 2001;24:587-9.

10. Hogan CJ, Ruland RT. High-pressure injection injuries to the upper extremity: a review of the literature. J Orthop Trauma 2006;20:503-11. 